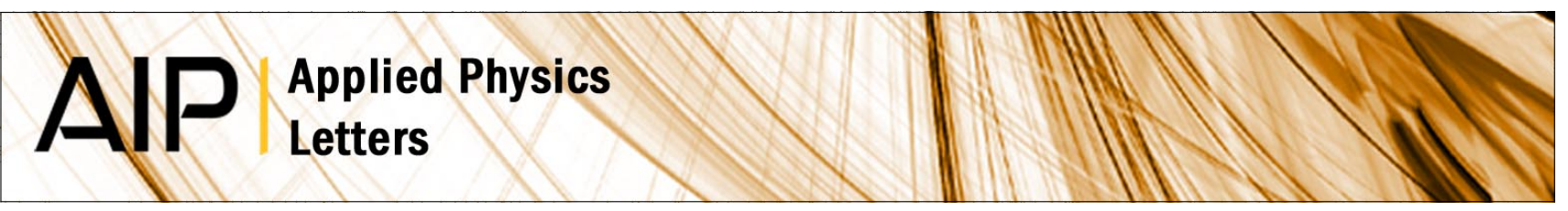

\title{
Generalized form of anhysteretic magnetization function for Jiles-Atherton theory of hysteresis
}
A. Raghunathan, Y. Melikhov, J.
E. Snyder, and D.
C. Jiles

Citation: Appl. Phys. Lett. 95, 172510 (2009); doi: 10.1063/1.3249581

View online: http://dx.doi.org/10.1063/1.3249581

View Table of Contents: http://apl.aip.org/resource/1/APPLAB/v95/i17

Published by the American Institute of Physics.

\section{Related Articles}

Piezoresponse force microscopy and vibrating sample magnetometer study of single phased Mn induced multiferroic BiFeO3 thin film

J. Appl. Phys. 111, 064110 (2012)

Tunable magnetocaloric effect near room temperature in La0.7-xPrxSr0.3MnO3 $(0.02 \leq x \leq 0.30)$ manganites J. Appl. Phys. 111, 063918 (2012)

Origin of magnetic properties and martensitic transformation of Ni-Mn-In magnetic shape memory alloys Appl. Phys. Lett. 100, 132402 (2012)

Ferromagnetic phase transition in zinc blende (Mn, $\mathrm{Cr}$ )S-layers grown by molecular beam epitaxy

Appl. Phys. Lett. 100, 132405 (2012)

Magnetic properties and thermal stability of (Fe,Co)-Mo-B-P-Si metallic glasses

J. Appl. Phys. 111, 063906 (2012)

\section{Additional information on Appl. Phys. Lett.}

Journal Homepage: http://apl.aip.org/

Journal Information: http://apl.aip.org/about/about_the_journal

Top downloads: http://apl.aip.org/features/most_downloaded

Information for Authors: http://apl.aip.org/authors

\section{ADVERTISEMENT}

\section{(@) ACCELERATE AMBER AND NAMD BY $5 X$. NVIDIA TRYIT ONA FREE, REMOTELYYHOSTED CLUSTER.}




\title{
Generalized form of anhysteretic magnetization function for Jiles-Atherton theory of hysteresis
}

\author{
A. Raghunathan, ${ }^{\text {a) }}$ Y. Melikhov, J. E. Snyder, and D. C. Jiles \\ Wolfson Centre for Magnetics, Cardiff University, Cardiff CF24 3AA, United Kingdom
}

(Received 8 April 2009; accepted 21 September 2009; published online 29 October 2009)

\begin{abstract}
A generalized form of anhysteretic magnetization function to extend Jiles-Atherton theory to different forms of anisotropy has been derived. The general equation for the function has been compared with those of calculations made on the basis of known equations for specific cases: axially anisotropic (one-dimensional), planar anisotropic (two-dimensional), and isotropic (three-dimensional). The Jiles-Atherton model using the proposed functional form of generalized anhysteretic magnetization function for anisotropy dependence has been validated and the necessary equations derived. It has been shown in this work that this functional form of anhysteretic magnetization with necessary boundary conditions can be reduced to the familiar specific model equations in the particular cases. (C) 2009 American Institute of Physics. [doi:10.1063/1.3249581]
\end{abstract}

In ferromagnetic materials, the magnetization path followed by the specimen changes with applied field. According to the Jiles-Atherton (JA) theory, ${ }^{1}$ the total magnetization, $M$, of a ferromagnetic material is a sum of contributions of irreversible, $M_{\text {irr }}$, and reversible, $M_{\text {rev }}$, magnetization components:

$$
M=M_{\text {irr }}+M_{\text {rev }} .
$$

In the model, $M_{\text {irr }}$ is attributed to domain wall pinning whereas $M_{\text {rev }}$ is attributed to reversible bowing of domain walls. Deriving these two magnetization components from micro-structural processes, the differential equation describing the dependence of magnetization, $M$, on magnetic field, $H$, can be constructed:

$$
\begin{aligned}
& M_{\mathrm{irr}}=M_{\mathrm{an}}-k \delta \frac{d M_{\mathrm{irr}}}{d H_{e}}, \\
& M_{\mathrm{rev}}=c\left(M_{\mathrm{an}}-M_{\mathrm{irr}}\right),
\end{aligned}
$$

where $M_{\text {an }}$ is the anhysteretic magnetization which is a function of energy of the moments in a domain and can have different forms depending on the anisotropy.

The effective field term, $H+\alpha M$, arises from the contributions of applied field and magnetic interaction between domains. The domain coupling, $\alpha$, and density, $a$, affect the slope of the hysteresis loop. The microstructural parameter $k$, being proportional to the pinning site density and energy, primarily determines the coercivity of the hysteresis loop. ${ }^{2}$ The reversibility factor $c$ is the representative of domain wall bowing. The directional parameter $\delta$ takes the value +1 when $H$ increases and -1 when $H$ decreases.

The JA theory has been previously extended to incorporate magnetoelastic ${ }^{3,4}$ and thermal effects. ${ }^{5,6}$ The previous works on extending JA model to include anisotropy ${ }^{7,8}$ were based on expressing anhysteretic magnetization in terms of known model equations shown later in Eqs. (4)-(6) for specific cases: axially anisotropic [so-called one-dimensional (1D)] with applied field along the easy axis, planar aniso-

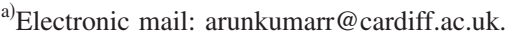

tropic [two-dimensional (2D)] with applied field in the easy plane, and isotropic [three-dimensional (3D)].

Anhysteretic function for an axially anisotropic material (1D case) with applied field along the easy axis, is

$$
M_{\text {an }}=M_{s}[\tanh (h)],
$$

for a planar anisotropic material (2D case) with applied field in the easy plane, ${ }^{10}$

$$
M_{\mathrm{an}}=M_{s}\left[\frac{\sum_{n=0}^{\infty} \frac{h^{2 n+1}}{(n+1) ! 2^{2 n+1}}}{1+\sum_{n=0}^{\infty} \frac{h^{2(n+1)}}{(n+1) ! 2^{2(n+1)}}}\right],
$$

and for isotropic materials (3D case), ${ }^{2}$

$$
M_{\text {an }}=M_{S}\left[\operatorname{coth}(h)-\frac{1}{h}\right],
$$

where $h=H_{e} / a, a=\left(k_{B} T\right) /\left(\mu_{0} m\right)$, and $k_{B}$ is the Boltzmann constant; $T$ is the temperature, $\mu_{0}$ is the permeability of vacuum, and $m$ is the magnetic moment of a typical domain.

As there is no known generalized form of anhysteretic magnetization derived so far, the hysteresis behavior of anisotropic or isotropic materials can be modeled only by solving the respective known anhysteretic magnetization function using JA theory. This work focuses on deriving a functional form of anhysteretic magnetization that would generalize the anisotropy dependence of JA theory and also, with necessary boundary conditions, be reduced to the specific known model equations in limiting cases given in Eqs. (4)-(6). In this work, the functional form is generalized to uniaxial anisotropy of arbitrary direction, which also includes easy plane. When subjected to an external field, each magnetic moment has a potential energy that tries to align it parallel to the applied field. This energy is given by

$$
E_{m}=-\mu_{0} m \cdot H_{e}=-\mu_{0} m H_{e} \cos \theta .
$$

The anisotropy energy, for uniaxial materials, can be written to lowest order as 


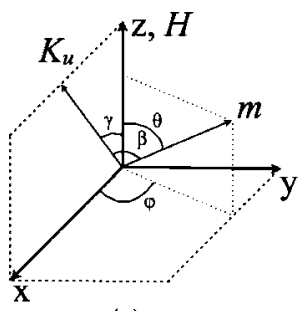

(a)

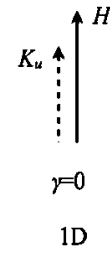

$1 \mathrm{D}$
2D

(b)

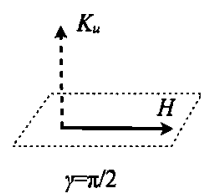

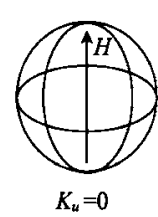

$3 \mathrm{D}$
FIG. 1. Representation of vectors in the spherical coordinate system. The field $H$ is applied along $Z$ direction, $K_{u}$ is the direction of anisotropy, $m$ is the moment direction, and angles $\theta$ and $\varphi$ are polar coordinates.

$$
E_{a}=K_{u} \sin ^{2} \beta
$$

where $K_{u}$ is the anisotropy constant, $\beta$ and $\theta$ are the angles made by magnetic moment with unique axis and applied field, respectively.

According to statistical thermodynamics, considering there is no direct interaction between the domains, the individual partition function can be represented as ${ }^{9}$

$$
\begin{aligned}
Z_{m}= & \sum_{\text {states }} \exp \left[\frac{-\left(E_{m}+E_{a}\right)}{k_{B} T}\right]=\sum_{\text {states }} \exp (h \cos \theta \\
& \left.-\kappa \sin ^{2} \beta\right),
\end{aligned}
$$

where $h$ and $\kappa$ are energy ratios and are given by $h=H_{e} / a$, $\kappa=K_{u} /\left(k_{B} T\right)$, and $a=\left(k_{B} T\right) /\left(\mu_{0} m\right)$. The interaction in this case depends on an indirect mean field coupling. The total partition function, $Z$, associated with $N$ independent moments is given in terms of individual partition functions by $\left(Z_{m}\right)^{N}$. The Gibbs free energy can be calculated from the total partition function as

$$
G=-k_{B} T \ln Z=-N k_{B} T \ln Z_{m} .
$$

The anhysteretic magnetization, $M_{\text {an }}$, which is a function of energy of $N$ moments occupying a volume $\Delta V$ in a domain, can be expressed as a change in Gibbs free energy with respect to the field at constant temperature, ${ }^{9}$

$$
M_{\mathrm{an}}=-\frac{1}{\mu_{0} \Delta V}\left[\frac{\partial G}{\partial H_{e}}\right]_{T} .
$$

To derive the functional form of anhysteretic magnetization, the partition function should be in a generalized form. Hence the generalized integral form of individual partition function, $Z_{m}$, based on spherical coordinate system shown in Fig. 1, is written as

$$
Z_{m}=\int_{0}^{2 \pi} \int_{0}^{\pi} \exp \left(h \cos \theta-\kappa \sin ^{2} \beta\right) \sin \theta d \theta d \varphi .
$$

Using the individual partition function shown in Eq. (12), the Gibbs free energy can be calculated from Eq. (10). The anhysteretic magnetization can then be derived from Gibbs free energy as

$$
\begin{aligned}
M_{\mathrm{an}}= & \frac{N k_{B} T}{\mu_{0} \Delta V} \frac{\partial}{\partial H_{e}}\left\{\operatorname { l n } \left[\int_{0}^{2 \pi} \int_{0}^{\pi} \exp (h \cos \theta\right.\right. \\
& \left.\left.\left.-\kappa \sin ^{2} \beta\right) \sin \theta d \theta d \varphi\right]\right\} .
\end{aligned}
$$

Substituting values of $h$ and $a$ in the above equation and taking the partial derivative we get,

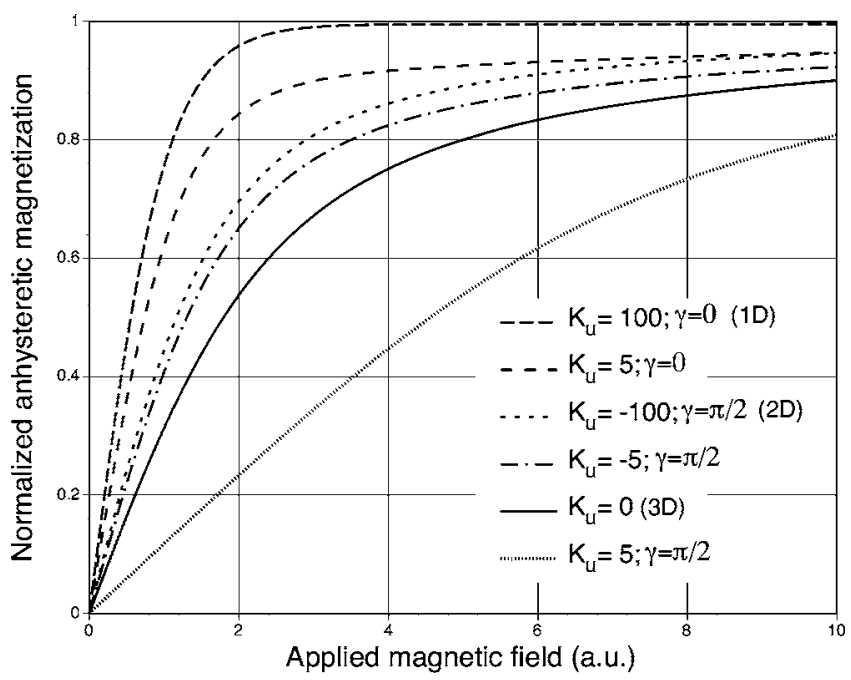

FIG. 2. Anhysteretic magnetization curves for various values of anisotropy constants numerically calculated from generalized functional form. The $\left(K_{u}>0, \gamma=0\right)$ represent uniaxial anisotropy with applied field along the easy axis, $\left(K_{u}<0, \gamma=\pi / 2\right)$ represents planar anisotropy with applied field in the easy plane, $K_{u}=0$ represents isotropy, and $\left(K_{u}>0, \gamma=\pi / 2\right)$ represents the hard direction anhysteretic magnetization when the applied field is orthogonal to the uniaxial easy axis.

$$
M_{\mathrm{an}}=M_{s} \frac{\int_{0}^{2 \pi} \int_{0}^{\pi} \exp \left(h \cos \theta-\kappa \sin ^{2} \beta\right) \sin \theta \cos \theta d \theta d \varphi}{\int_{0}^{2 \pi} \int_{0}^{\pi} \exp \left(h \cos \theta-\kappa \sin ^{2} \beta\right) \sin \theta d \theta d \varphi},
$$

where $M_{s}$ is the spontaneous magnetization and is given by $M_{s}=(N m) /(\Delta V)$.

In order to generalize the anhysteretic magnetization function shown in Eq. (13), the angle made by moments with anisotropy axis, $\beta$, should be represented in terms of other angle parameters. This can be achieved by defining the direction cosines of $K_{u}(\sin \gamma, 0, \cos \gamma)$ and $m(\sin \theta \cos \varphi, \sin \theta \sin \varphi, \cos \theta)$ from the spherical coordinate system (see Fig. 1). The scalar product yields $m \cdot K_{u}$ $=|m|\left|K_{u}\right| \cos \beta$, which leads to

$$
\cos \beta=\sin \gamma \sin \theta \cos \varphi+\cos \gamma \cos \theta .
$$

Hence, the generalized functional form of anhysteretic magnetization, shown in Eq. (13) can be rewritten using a trigonometric identity as

$M_{\text {an }}=M_{s} \frac{\int_{0}^{2 \pi} \int_{0}^{\pi} \exp \left(h \cos \theta-\kappa\left[1-\cos ^{2} \beta\right]\right) \sin \theta \cos \theta d \theta d \varphi}{\int_{0}^{2 \pi} \int_{0}^{\pi} \exp \left(h \cos \theta-\kappa\left[1-\cos ^{2} \beta\right]\right) \sin \theta d \theta d \varphi}$.

This functional form can be validated against specific model equations shown in Eqs. (4)-(6) by applying the necessary boundary conditions for specific cases: axially anisotropic, planar anisotropic, and isotropic.

Consider first that the anisotropy is positive and the applied field is parallel to the anisotropic easy direction, i.e., $\gamma=0$. In the case of extremely high anisotropy, this case can be reduced to a 1D problem as shown in Fig. 2. On substitution of the boundary condition $(\gamma=0)$ in the generalized functional form given in Eq. (15), we get 
$M_{\mathrm{an}}=M_{s} \frac{\int_{0}^{2 \pi} \int_{0}^{\pi} \exp \left(h \cos \theta-\kappa\left[1-\cos ^{2} \theta\right]\right) \sin \theta \cos \theta d \theta d \varphi}{\int_{0}^{2 \pi} \int_{0}^{\pi} \exp \left(h \cos \theta-\kappa\left[1-\cos ^{2} \theta\right]\right) \sin \theta d \theta d \varphi}$.

Integrating with respect to $\varphi$ and introducing a new variable $x=\cos \theta$, the second order polynomial in the exponent can be rearranged, enabling us to write both numerator and denominator terms in the form of Dawson's integral, ${ }^{11}$

$$
F(z)=\exp \left(-z^{2}\right) \int_{0}^{z} \exp \left(t^{2}\right) d t
$$

and on simplification we get,

$$
M_{\mathrm{an}}=M_{s} \frac{\left[[\exp (2 h)-1] \sqrt{\kappa}+h F\left(\frac{h-2 \kappa}{2 \sqrt{\kappa}}\right)-\exp (2 h) h F\left(\frac{h+2 \kappa}{2 \sqrt{\kappa}}\right)\right]}{2 \kappa\left[-F\left(\frac{h-2 \kappa}{2 \sqrt{\kappa}}\right)+\exp (2 h) F\left(\frac{h+2 \kappa}{2 \sqrt{\kappa}}\right)\right]} .
$$

From the asymptotic expansion of Dawson's integral, ${ }^{11}$ for large $|z|$, the function $F(z) \approx 1 /(2 z)$. Applying the above boundary condition in Eq. (18),

$$
M_{\mathrm{an}}=\lim _{\kappa \rightarrow \infty} M_{s} \frac{\left[[\exp (2 h)-1] \sqrt{\kappa}+h\left(\frac{\sqrt{\kappa}}{h-2 \kappa}\right)-\exp (2 h) h\left(\frac{\sqrt{\kappa}}{h+2 \kappa}\right)\right]}{2 \kappa\left[-\left(\frac{\sqrt{\kappa}}{h-2 \kappa}\right)+\exp (2 h)\left(\frac{\sqrt{\kappa}}{h+2 \kappa}\right)\right]} .
$$

Neglecting higher order terms, rearranging and applying limits we get,

$$
M_{\mathrm{an}} \approx M_{s}[\tanh (h)] \text {. }
$$

This solution is same as in Eq. (3). Hence this shows that the new generalized function can reduce to the known form for specific examples, such as the uniaxial case.

Now, consider that the anisotropy is negative and the applied field is perpendicular to the anisotropy direction, i.e., $\gamma=\pi / 2$. As shown in Fig. 1(b), the preferred direction in this case is a plane. Hence this case can be reduced to a $2 \mathrm{D}$ problem in case of the extremely high negative anisotropy. Substituting the condition $\gamma=\pi / 2$ in Eq. (14), we get

$M_{\mathrm{an}}=M_{s} \frac{\int_{0}^{2 \pi} \int_{0}^{\pi} \exp \left(h \cos \theta-\kappa\left[1-\sin ^{2} \theta \cos ^{2} \varphi\right]\right) \sin \theta \cos \theta d \theta d \varphi}{\int_{0}^{2 \pi} \int_{0}^{\pi} \exp \left(h \cos \theta-\kappa\left[1-\sin ^{2} \theta \cos ^{2} \varphi\right]\right) \sin \theta d \theta d \varphi}$.

Rearranging Eq. (21) and applying trigonometric identities in order to integrate with respect to $\varphi$ we get,

$$
M_{\mathrm{an}}=M_{s} \frac{\int_{0}^{\pi} \exp \left(h \cos \theta-\frac{\kappa}{4}(3+\cos 2 \theta)\right) \sin 2 \theta\left[\int_{0}^{2 \pi} \exp \left(\frac{\kappa}{2} \sin ^{2} \theta \cos 2 \varphi\right) d \varphi\right] d \theta}{2 \int_{0}^{\pi} \exp \left(h \cos \theta-\frac{\kappa}{4}(3+\cos 2 \theta)\right) \sin \theta\left[\int_{0}^{2 \pi} \exp \left(\frac{\kappa}{2} \sin ^{2} \theta \cos 2 \varphi\right) d \varphi\right] d \theta} .
$$

To evaluate the integral with respect to $\varphi$, Sonine's expansion for modified Bessel function ${ }^{10,11}$ was used,

$$
\exp (y \cos \varphi)=I_{0}(y)+2 \sum_{p=1}^{\infty} I_{p}(y) \cos (p \varphi),
$$

where $I(y)$ are modified Bessel functions of first kind. As the contributions of higher order $(p \neq 0)$ Bessel functions to Eq. (23) are negligible, ${ }^{10}$ only $I_{0}(y)$ is considered. Substituting
Eq. (23) into Eq. (22) and integrating with respect to $\varphi$ will yield,

$$
M_{\mathrm{an}}=M_{s} \frac{\int_{0}^{\pi} \exp \left(h \cos \theta-\frac{\kappa}{4}[3+\cos 2 \theta]\right) I_{0}\left(\frac{\kappa}{2} \sin ^{2} \theta\right) \sin \theta \cos \theta d \theta}{\int_{0}^{\pi} \exp \left(h \cos \theta-\frac{\kappa}{4}[3+\cos 2 \theta]\right) I_{0}\left(\frac{\kappa}{2} \sin ^{2} \theta\right) \sin \theta d \theta} .
$$

The procedure to solve this integral is similar to the one shown by Jiles et al. ${ }^{10}$ The solution given in Eq. (4). has no closed form but can be solved numerically to yield hard axis anhysteretic curve of planar anisotropic materials.

When there is no preferred direction in a material, that is anisotropy is zero, i.e., $K_{u}=0$ and hence $\kappa=0$, this case has three-dimensional solution as shown in Fig. 2. On substitution of boundary condition in Eq. (15), we get

$$
M_{\mathrm{an}}=M_{s} \frac{\int_{0}^{2 \pi} \int_{0}^{\pi} \exp (h \cos \theta) \cos \theta \sin \theta d \theta d \varphi}{\int_{0}^{2 \pi} \int_{0}^{\pi} \exp (h \cos \theta) \sin \theta d \theta d \varphi} .
$$

Solving the above integral yields Langevin's function and is given by

$$
M_{\text {an }}=M_{s}\left[\operatorname{coth}(h)-\frac{1}{h}\right] .
$$

This solution is the same as for the isotropic case, shown in Eq. (6). This Langevin's function gives the anhysteretic magnetization of isotropic materials.

As an example, the plots of anhysteretic magnetization for various values of $K_{u}$ and $\gamma$ are shown in Fig. 2. These plots were obtained by numerically solving the generalized functional form, shown in Eq. (15), for various values of $K_{u}$ and $\gamma$. The anisotropy energy, shown in Eq. (8), can also be extended for the easy cone or the multiaxial case by considering more than one anisotropy constant.

In this work, the JA theory was extended to incorporate anisotropy by deriving a generalized functional form of the anhysteretic magnetization. It was shown that the derived generalized functional form, with necessary boundary conditions, can be reduced to the known specific model equations for specific cases: axially anisotropic (1D), planar anisotropic (2D), and isotropic (3D).

This research was supported by the UK EPSRC (EP/ D057094) and the US NSF (DMR-0402716).

${ }^{1}$ D. C. Jiles and D. L. Atherton, J. Magn. Magn. Mater. 61, 48 (1986).

${ }^{2}$ D. C. Jiles, Introduction to Magnetism and Magnetic Materials, 2nd ed. (Chapman and Hall, London, 1991).

${ }^{3}$ M. J. Sablik and D. C. Jiles, IEEE Trans. Magn. 29, 2113 (1993).

${ }^{4}$ D. C. Jiles, J. Phys. D: Appl. Phys. 28, 1537 (1995).

${ }^{5}$ P. R. Wilson, J. N. Ross, and A. D. Brown, IEEE Trans. Power Electron. 17, 55 (2002)

${ }^{6}$ P. Andrei, A. Stancu, H. Hauser, and P. Fulmek, J. Optoelectron. Adv. Mater. 9, 1137 (2007).

${ }^{7}$ Y. M. Shi, D. C. Jiles, and A. Ramesh, J. Magn. Magn. Mater. 187, 75 (1998)

${ }^{8}$ D. C. Jiles and J. B. Thoelke, J. Magn. Magn. Mater. 134, 143 (1994).

${ }^{9}$ G. Bertotti, Hysteresis in Magnetism (Academic, London, 1998).

${ }^{10}$ D. C. Jiles, S. J. Lee, J. Kenkel, and K. L. Metlov, Appl. Phys. Lett. 77, 1029 (2000).

${ }^{11}$ C. M. Bender and S. A. Orszag, Advanced Mathematical Methods for Scientists and Engineers (McGraw-Hill, London, 1978). 